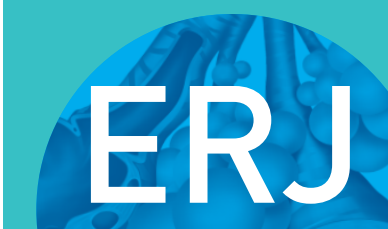

open research
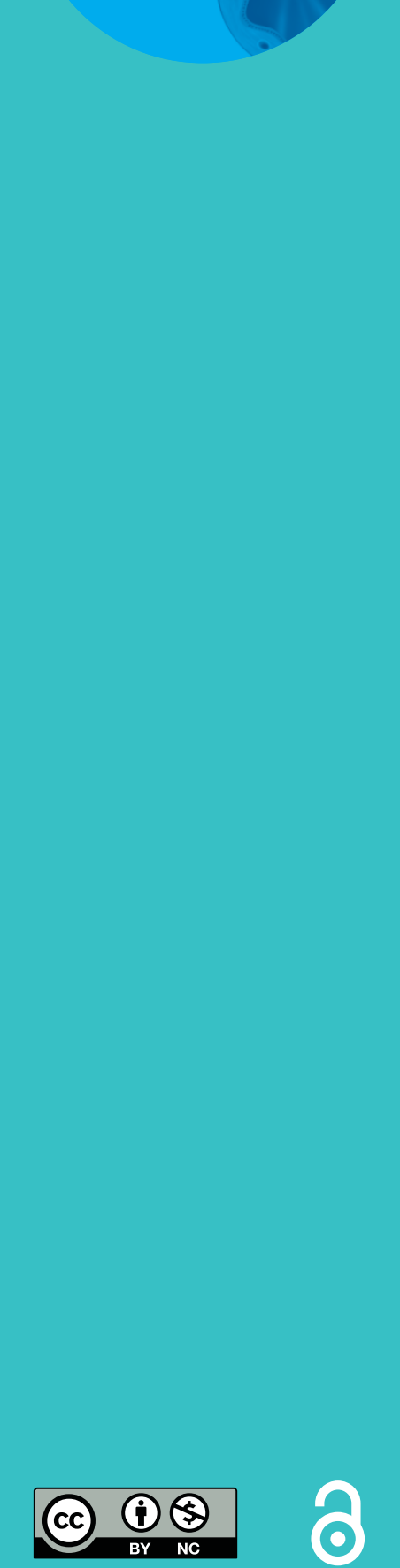

\section{European Respiratory Society International Congress 2018: four shades of epidemiology and tobacco control}

\author{
Maaike de Vries (10 ${ }^{1,2}$, Eleanor L. Axson $\mathbb{1}^{3}$, Jate Ratanachina ${ }^{3,4,5}$, \\ Orianne Dumas $\mathbb{1}^{6,7}$, Sara De Matteis ${ }^{3}$, Annabelle Bédard ${ }^{8,9,10}$, \\ Célina Roda $8,9,10,11$, Subhabrata Moitra $8,9,10$, Elif Dagli ${ }^{12}$, Athina Diamanti ${ }^{13,14}$, \\ Asli Gorek Dilektasli ${ }^{15}$, Sofia Ravara ${ }^{16,17,18}$ and André F.S. Amaral (1) ${ }^{3}$
}

Affiliations: ${ }^{1}$ University of Groningen, University Medical Centre Groningen, Dept of Epidemiology, Groningen, The Netherlands. ${ }^{2}$ University of Groningen, University Medical Centre Groningen, Groningen Research Institute for Asthma and COPD (GRIAC), Groningen, The Netherlands. ${ }^{3}$ Population Health and Occupational Disease, National Heart and Lung Institute, Imperial College London, London, UK. ${ }^{4}$ King Chulalongkorn Memorial Hospital, Thai Red Cross Society, Bangkok, Thailand. ${ }^{5}$ Dept of Preventive and Social Medicine, Chulalongkorn University, Bangkok, Thailand. 'INSERM, U1168, VIMA: Aging and chronic diseases, Epidemiological and Public Health Approaches, Villejuif, France. ${ }^{7}$ Universite de Versailles St-Quentin-en-Yvelines, UMR-S 1168 , Montigny le Bretonneux, France. ${ }^{8}$ ISGlobal, Barcelona, Spain. ${ }^{9}$ Universitat Pompeu Fabra (UPF), Barcelona, Spain. ${ }^{10}$ CIBER Epidemiología y Salud Pública (CIBERESP), Barcelona, Spain. ${ }^{11}$ INSERM U1153, HERA team: Health Environmental Risk Assessment, Paris Descartes University, Paris Faculty of Pharmacy, Paris, France. ${ }^{12}$ Marmara University, Istanbul, Turkey. ${ }^{13}$ Midwifery Department of University of West Attica, Athens, Greece. ${ }^{14}$ Dept of Critical Care and Pulmonary Services, University of Athens Medical School, Evangelismos Hospital, Athens, Greece. ${ }^{15}$ Uludag University, Faculty of Medicine, Dept of Pulmonary Medicine, Bursa, Turkey. ${ }^{16}$ Health Sciences Research Centre (CICS-UBI), Faculty of Health Sciences, University of Beira Interior, Covilha, Portugal. ${ }^{17}$ Public Health Research Centre, National School of Public Health, NOVA University, Lisbon, Portugal. ${ }^{18} \mathrm{CHUCB}$, University Hospital, Covilha, Portugal.

Correspondence: André F.S. Amaral, Population Health and Occupational Disease, National Heart and Lung Institute, Imperial College London, Emmanuel Kaye Building, 1B Manresa Road, London SW3 6LR, UK. E-mail: a.amaralaimperial.ac.uk

ABSTRACT In this article, early career members and experienced members of the Epidemiology and Environment Assembly of the European Respiratory Society highlight and summarise a selection of six sessions from the Society's annual congress, which in 2018 was held in Paris, France. The topics covered in these sessions span from cutting-edge molecular epidemiology of lung function to clinical, occupational and environmental epidemiology of respiratory disease, and from emergent tobacco products to tobacco control.

@ERSpublications

Highlights of \#ERSCongress 2018 from @ERStalk Assembly 6 http://ow.ly/O03v30nEHhJ

Cite this article as: de Vries M, Axson EL, Ratanachina J, et al. European Respiratory Society International Congress 2018: four shades of epidemiology and tobacco control. ERJ Open Res 2019; 5: 00217-2018 [https://doi.org/10.1183/23120541.00217-2018].

Copyright $\odot$ ERS 2019. This article is open access and distributed under the terms of the Creative Commons Attribution Non-Commercial Licence 4.0. 


\section{Multiomic studies in epidemiology: what can they tell us?}

The increasing use of multiomics in the field of the molecular epidemiology of lung health was nicely illustrated during this session. Covering both asthma and chronic obstructive pulmonary disease (COPD), the studies presented during this session showed novel findings on the genetics of these diseases using innovative genomic approaches including genetic risk scores (GRS), genome-wide interaction (GWI) studies and identification of rare genetic variants.

The usefulness of a GRS for COPD recently reported by WAIN et al. [1] was demonstrated by two speakers. WAIN [2] presented an extended version of her COPD GRS with 139 novel signals and a weighted risk score based on 279 variants. The weighted risk score was significantly associated with risk of COPD, with an odds ratio of 1.55 (95\% CI 1.47-1.63) for each standard deviation of the risk score. Using data from the SPIROMICS study, Elizabeth Oelsner reported that the GRS from WaIN et al. [1] was not only associated with lung function, but also with computed tomography (CT) scan measures such as lung density, spatially matched airway dimensions and small airway counts [3]. In addition, she suggested that variation in lung structure could be an important mediator of genetic risk factors underlying COPD. Interestingly, Natalie Terzikhan showed that the diffusing capacity of the lung for carbon monoxide (DLCO) may also be heritable and is significantly associated with a genetic variant in GPR126 [4]. Terzikhan reported that the expression of this gene was decreased in lung tissue of patients with COPD, but whether GPR126 has a functional role in COPD is unclear.

Three studies focused on the aetiology of asthma but used different approaches to improve our understanding of the development of this disease. Emmanuelle Bouzigon reported on the findings of a meta-analysis of five GWI studies combined with survival analysis carried out to identify genetic variants that interact with sex and associate with time to asthma onset. One female-specific and two male-specific variants were identified, all involved in inflammatory processes and immune cell regulation [5]. Cosetta Minelli showed results from a Mendelian randomisation study using UK Biobank data and provided evidence that early puberty has detrimental effects on asthma [6]. As these effects were observed in both sexes, it is unlikely that female sex hormones act as mediators of this causal association. Using data from the Avon Longitudinal Study of Parents and Children cohort, and after assessing the association between maternal dietary antioxidant intake during pregnancy and childhood asthma [7], Annabelle Bédard reported that maternal intake of zinc might be associated with better lung function of the offspring.

The last two speakers of this session presented results from studies that aimed to identify rare genetic variants associated with COPD and asthma. Maaike de Vries selected rare genetic variants that were predicted to be pathogenic and present in at least three individuals within a small homologous population of 36 never-smokers with COPD. Two of these rare genetic variants in the genes ARAP3 and KIF27 were associated with COPD in two general population-based cohorts (LifeLines and the Rotterdam Study) [8]. Finally, Diana Chang, who looked at common and rare variant burden GWAS in a large group of people of European ancestry living with asthma, reported a novel variant in THSD4 associated with asthma risk. In addition, she showed a significant enrichment of genes implicated in primary ciliary dyskinesia with asthma risk among patients with low eosinophil counts [9].

What is the clinical relevance of these findings? What do they tell us about the underlying mechanisms of COPD and asthma? For now, these questions remain unanswered but they do give clues and generate hypotheses for further testing, eventually in epidemiological studies combining genetics with epigenetics, transcriptomics and other 'omics of respiratory disease.

\section{COPD and chronic respiratory disease: from misclassification to radiological findings}

The research presented in this session highlighted great advances in the understanding of chronic respiratory diseases, and the growing complexity and systemically entangled nature of their prevention, morbidity and management.

BACKMAN et al. [10] investigated the prevalence of COPD in Sweden following decades of smoking decline using data from the West Sweden Asthma Study and the Obstructive Lung Disease in Northern Sweden study. Backman reported a decrease in the prevalence of COPD from $11-17 \%$ to $5.6-8.4 \%$, a noticeable change since the turn of the millennium. As cigarette smoking is the main risk factor for COPD [11], she suggested that smoking decline in Sweden over the last decades is the main driving force behind this decline in the prevalence of COPD.

Several polymorphisms of the FAM13A gene have previously been associated with increased lung function decline in smokers [12]. Using data from the Korean Genome Epidemiology Study, KIM et al. [13] tracked lung function decline in 2900 male current and former smokers over 16 years. In this study, participants had been genotyped for the single-nucleotide polymorphism rs3756050 of FAM13A. Kim reported that 
while all former smokers benefited from reduced lung function decline, former smokers homozygous for the nonrisk allele experienced significantly less lung function decline compared to those with at least one risk allele.

Not smoking is perhaps the most effective means of COPD prevention but other lifestyle factors may also influence risk. Recent research has suggested that increased fruit and vegetable intake is associated with decreased risk of COPD in current and former smokers [14]. METERAn et al. [15] used data on 11458 adults from the Danish Twin Registry to investigate whether this association was due to underlying genetic factors. Meteran showed that increasing self-reported intake of fruit and raw vegetables was significantly associated with decreased risk of COPD. Whether this association is explained by genetic factors is difficult to say since results from the cotwin analysis, designed to determine the influence of genetics, were inconclusive.

The relationship between chronic respiratory diseases and cardiovascular diseases may be mediated by inflammation [16], which may activate coagulation. Yet, little is known about the role of coagulants in chronic respiratory diseases such as COPD. Gunnar Husebø presented findings from work he and his colleagues carried out using data from the Bergen COPD Cohort Study. The aim was to investigate coagulation markers as potential biomarkers for acute exacerbations of chronic obstructive pulmonary disease (AECOPD) [17]. They found that thrombin-antithrombin (TAT) complex, activated protein $\mathrm{C}$-protein $\mathrm{C}$ inhibitor and D-dimer were all at significantly higher concentrations during AECOPD than in the stable state. Additionally, higher TAT in stable state was associated with an increased AECOPD frequency, while higher D-dimer was associated with increased mortality.

Logical fallacies resultant from a lack of research are easily made when attempting to manage patients with multimorbidity due to shear complexity and the desire to do no harm. Fears that $\beta$-blockers would increase bronchoconstriction led to their underuse in COPD patients; however, numerous studies have now shown that $\beta$-blockers are safe [16]. RASMUSSEN et al. [18] examined nationwide $\beta$-blocker prescribing practices post-myocardial infarction in Denmark from 1995-2015. They found that $\beta$-blockers are still systematically underused in COPD patients but that their utilisation has increased over time.

Morbidity and mortality due to chronic respiratory diseases is a significant public health burden [19, 20]. Nils Hoyer presented findings from a study where, using data from the Danish Lung Cancer Screening Trial, he and colleagues investigated the most likely causes of hospital admission in smokers with interstitial lung abnormalities (ILAs) [21]. Hoyer reported that those with ILA were more likely to be admitted to hospital than those without ILA, and that the most common diagnoses resulting from these admissions were respiratory (COPD, pneumonia, lung abscess and interstitial lung disease), cardiovascular (heart failure, pulmonary embolism and peripheral vascular disease) and malignant (lung cancer) disease. GAYLE et al. [22] examined causes of death in chronic respiratory disease patients from the Clinical Practice Research Datalink in the UK. Gayle reported that from 2005 to 2014, while deaths due to circulatory diseases decreased, deaths due to respiratory diseases remained constant. These trends may reflect how, unlike circulatory diseases, respiratory medicine has not benefited from the development of disease-modifying interventions.

\section{Occupational and environmental lung diseases: from early influences and novel exposures to preventive approaches}

This session brought together speakers from Europe, North America and Africa to talk about respiratory diseases and their links mainly to occupational exposures. It covered studies of several diseases on several continents, the use of media to increase awareness of occupational disease and guidelines on how to deal with occupational asthma.

Sara de Matteis presented the results of an update of the British Occupational Health Research Foundation guidelines on occupational asthma [23]. De Matteis performed a systematic review, qualitatively appraised using the Grading of Recommendations, Assessment, Development and Evaluations scoring system, and produced evidence-based statements and recommendations for all stakeholders. She reported that the occupational asthma incidence (1.6-300 cases per million workers) has remained stable over time and that the best occupational asthma prevention intervention is to control exposure to potential asthmagens in the workplace. Serial peak expiratory flow measurements remain the recommended approach for occupational asthma diagnosis, and early identification of cases via health surveillance and exposure avoidance to the causal agents remain pivotal to cure or at least improve occupational asthma prognosis. De Matteis also reported that, based on the systematic review, being a cleaner increases the risk of occupational asthma [23]. A relatively large and recent study of 2030 US healthcare workers supports this finding [24]. Paul Henneberger reported that workers who were in charge of cleaning fixed surfaces were almost twice as likely to have current asthma as those who do not carry out cleaning tasks. Administration of aerosolised 
medications was also associated with current asthma and asthma exacerbations. Individuals with allergic diseases in childhood may have increased risk of work-related asthma in adulthood and would constitute a relevant target group for specific interventions, including career advice.

The influence of asthma and allergies in childhood in avoiding occupational exposure in early adulthood was examined in the prospective Melbourne Atopic Cohort Study, which included 363 participants with high risk of asthma and allergies [25]. Orianne Dumas reported that there was no evidence of avoidance of potentially hazardous exposures in young adults with a history of asthma or allergies, supporting the need for improved counselling regarding career choices.

Using data from a small study of 68 former welding, plumbing and heating apprentices in Quebec, Canada, Eva Suarthana examined the association between exposure to welding fumes and occupational rhinitis 7-17 years post-apprenticeship [26]. Several methods were used to evaluate both exposure (self-report, expert assessment and job-exposure matrix) and rhinitis (self-reported symptoms and peak nasal inspiratory flow). Suarthana reported that although workers exposed to welding fumes seemed to be at higher risk of rhinitis, the size of the study sample and the lack of statistical significance precluded any firm conclusions.

People who work fitting insulation in buildings are a population at risk of both respiratory and cardiovascular diseases, with asbestos as the most common occupational cause of morbidity and mortality among this population. A longitudinal study of 301 never-smoking unionised insulators in Alberta, Canada, showed that $54 \%$ of the insulators are exposed to asbestos. Samineh Kamravaei reported that insulators showed a lower COPD Assessment Test score (mean $4 \pm 4$ versus $6 \pm 5, \mathrm{p}<0.001$ ) and more lung abnormalities on chest radiography $(10 \%$ versus $3 \%, \mathrm{p}<0.01)$ than controls (police officers). In addition, the Framingham risk score (cardiovascular risk) in insulators older than 50 years was worse than that of same-age controls [27].

Dust from artificial stone, which is widely used worldwide, especially in kitchen work surfaces, has been a concern due to its ultrafine particles penetrating deeply in lungs. Noa Ophir presented the findings of a study in 100 Israeli workers exposed to artificial stone dust for up to 20 years. He reported that a decline in lung function values (total lung capacity, forced expiratory volume in $1 \mathrm{~s}$ (FEV1) and single-breath DLCO), a worsening CT score [28], and elevated levels of inflammatory cytokines (interleukin-6, interleukin-8 and tumour necrosis factor- $\alpha$ ) [29] were associated with a greater number of nano-range particles in induced sputum.

National administrative data can be useful in occupational lung disease epidemiology and intervention planning. Jill Murray reviewed the Pathology Automation computerised database recording South Africa miners' pathological findings from autopsy since 1975 [30], combined with demographic and clinical data. Murray reported that amongst 11901 records, 72\% worked in gold mining. Amongst black miners, silicosis and pulmonary tuberculosis (TB) prevalence increased over the period from 1975 to 2016. Employment duration, increasing age and autopsy year related to silicosis trends, whilst pulmonary TB was mainly affected by silicosis and HIV infection [31].

The European Lung Foundation, in collaboration with the European Respiratory Society (ERS), has developed an online tool that may help in improving awareness of occupational respiratory diseases in at-risk populations [32]. This tool was designed for members of the public to assess whether their occupation may pose a risk to their lung health (http://yourlungsatwork.europeanlung.org/en/). According to Emma Thompson, users invited through a social media advertising campaign targeting at-risk workers were asked to answer a quiz online, including six questions on respiratory symptoms and their link with work. Among $>8000$ participants, $89 \%$ were advised to talk to a healthcare professional after taking the quiz and $10 \%$ reported that they had to leave or change their job in the past because of their breathing.

\section{Effect of environmental exposure on lung function outcomes}

In this session, results from several high-quality cohort studies examining the effects of tobacco smoke and air pollution exposures on lung function were presented.

Elizabeth Oelsner [33] studied the effect of former and light smoking on lung function changes over time in US population-based cohorts. Oelsner reported that lung function decline was faster among former and light smokers compared to never-smokers, the effect being greater among the current light smokers.

Three longitudinal studies conducted in northern European countries, where pollutant concentrations are relatively low (i.e. average below European Union recommendations), were presented and all suggested adverse long-term effects of air pollution exposure on respiratory health [2-4]. Ane Johannessen presented findings from the Respiratory Health in Northern Europe study [34], where she and her colleagues investigated the effects of annual average concentrations of particulate matter (PM2.5 and PM10) and 
nitrogen dioxide on all-cause/respiratory sick leave 10 and 20 years later. PM2.5 and PM10 were found to be associated with all-cause sick leave and respiratory-sick leave 20 years later, but not 10 years later. In an urban Swedish population, Hanne Carlsen investigated the chronic effect of black carbon, PM2.5 and PM10 from different sources and found that traffic-related air pollution, particularly exposure to black carbon, was associated with low forced vital capacity (FVC) and FEV1 [35]. Childhood as a critical window of exposure was considered by Ingrid Kuiper. She and her co-workers investigated long-term effects of exposure to PM2.5, PM10, $\mathrm{NO}_{2}$ and greenness in childhood on respiratory health in adulthood in the RHINESSA cohort (Respiratory Health in Northern Europe, Spain and Australia) and showed that early-life exposure to outdoor air pollution was associated with respiratory symptoms and late-onset asthma in adulthood while those who resided closely to a greenspace during childhood had fewer symptoms in adulthood [36].

Body composition is known to be associated with lung function decline [37-39]. However, whether distinct body mass index (BMI) trajectories over time affect lung function decline is still unknown. Gabriela Peralta identified different BMI trajectories during adulthood and examined their associations with lung function decline in the European Community Respiratory Health Survey cohort [40]. Adults who remained overweight or obese throughout the study and those who became overweight were found to have greater lung function decline than those with a "normal" BMI throughout.

Most epidemiological studies have focused on single exposure-health effect relationships but the "exposome" approach has attracted growing interest and has been leading to new ways of analysing multiple exposures [41]. In the large European Human Early-Life Exposome cohort study, Valerie Siroux and colleagues conducted the first exposome study on lung function in children [42]. Using data from 1200 mother-child pairs, they assessed 85 prenatal and 125 postnatal exposures (biomonitoring data, geospatial data, sensor data, child health outcomes and 'omics signatures) and examined their effects on FEV1. Siroux reported that some previously known associations, such as those between exposure to phthalates and perfluoroalkoxyalkanes and FEV1 decline, were observed [42].

There is some evidence that the oral microbiota may influence respiratory health, especially in oral and dental conditions such as periodontal disease [43-45]. In adults from the RHINESSA study, Oistein Svanes identified 147 bacteria genera in the gingival fluid, and found associations between known oral and lung pathogens and lower lung function [46], supporting the hypothesis that oral bacteria may perturb pulmonary health either by entering the lungs through microaspiration or by infusing inflammatory mediators from inflamed gingival epithelium to the lungs.

The use of a fixed cut-off for the FEV1/FVC ratio to define airflow limitation rather than the lower limit of normal (LLN) may cause underdiagnosis among younger individuals [47], an issue that has been poorly studied. Yunus Çolak investigated whether underdiagnosed airflow limitation in young and middle-aged adults from the Copenhagen General Population Study was associated with a poorer prognosis [48], and found that individuals diagnosed with airflow limitation based on the LLN but not the fixed ratio at baseline had an increased risk of respiratory and cardiovascular morbidity and early death during follow-up.

The findings presented in this session highlight the importance of using a long-term approach when investigating the role of environmental or lifestyle factors, as well as the importance of using appropriate clinical parameters to diagnose airflow limitation.

Innovative tobacco cessation approaches and trends in tobacco and e-cigarette use Stopping smoking is challenging. However, evidence-based treatment delivered by trained healthcare providers (HCPs), combining both behavioural counselling and pharmacotherapy, is effective in helping smokers to achieve sustained abstinence [49]. Nevertheless, recent population-based surveys among European countries reveal a decreasing trend of assisted quitting with the support of HCPs towards unassisted quitting or the use of e-cigarettes [50]. In this session, three studies applying distinct approaches to this topic were presented. Paraskevi Katsaounou reported on the results of a World Health Organization (WHO)-ERS project on smoking cessation in primary care, which targeted three countries with a high prevalence of tobacco use and low availability of cessation services (i.e. Bangladesh, Greece and Moldova) [51]. The aim was to strengthen national health systems for treating tobacco dependence, especially in primary care, by establishing a national network of HCPs adequately trained in smoking cessation advice. This was achieved by engaging local government and health authorities, with the support of ERS and WHO experts, to deliver "train the trainers" workshops in cessation brief advice. The preliminary results of the first year show both wide reach and effectiveness, enrolling $>10000$ smokers in the three countries, and achieving successful high abstinence rates at 1 month (31.7\% to 48.4\%) [51, 52]. This project also highlights the win-win opportunity for partnerships in tobacco control, maximising 
resources and expertise, implementing the broad reach and most cost-effective clinical interventions, and supporting those countries with the most need. VAN SCHAYCK et al. [53] used a cluster randomised trial to investigate the impact of financial incentives offered through a workplace-based smoking cessation programme implemented in 61 companies in the Netherlands (640 smokers). The smokers in the intervention arm attended seven 90 -min cessation counselling sessions and received three $€ 50$ incentives: immediately after completing the programme, and 3 and 6 months later. Those in the control arm were provided with the counselling sessions but not the financial incentives. Van Schayck reported that the proportion of quitters was higher in the intervention arm, especially in the long term. This is a successful example of both health-promoting working places and real-world smoking cessation programmes, using the strategies of the Ottawa Charter [54], i.e. strengthening community actions and creating supportive environments. Finally, a randomised clinical trial carried out in Ireland assessed the effectiveness of the empirical, widely used and nonvalidated Allen Carr's Easyway to Stop Smoking method [55], which does not include pharmacotherapy [56]. The main aim of the study (300 smokers) was to assess whether this method, delivered in a group setting, would achieve higher abstinence rates than the Irish online Quit programme developed by the Irish National Health Services (www.quit.ie). Sheila Keogan reported that the Allen Carr method achieved significantly higher quit rates, both in the short and long terms. Further research on this method in other populations is recommended.

In this session, trends in tobacco and e-cigarette use from population-based surveys in Sweden, France and Russia were also presented. Emma Goksor reported that while in 2008, a postal questionnaire-based survey observed an alarming increase in young female smoking rates, in 2016, a repeat survey shows a decreasing trend in the same age group (16-25 years) from $20.5 \%$ to $15.2 \%$ [57]. Additionally, the sex gap in smoking prevalence has narrowed over time. However, the study observed that current smokers, in comparison to those interviewed in 2008, and females, in comparison to males, reported respiratory symptoms more often [57]. This trend is in line with recent research and points out that women may be more vulnerable than men to tobacco health hazards, and that cigarettes became more toxic over time [58]. Linnea Hedman presented findings from a study on use of e-cigarettes by schoolchildren carried out in Northern Sweden [59]. She reported that the prevalence of current smoking among boys and girls was $\sim 10 \%$. Current e-cigarette use was more common in boys, ever-smokers and snus users, and less common among those with a healthy diet. Although use of e-cigarettes was more common among ever-users of any tobacco product, $39 \%$ of current e-cigarette users at age 19 years were nonsmokers, which suggests that e-cigarettes are substituting traditional cigarettes among youth. Emeline Lequy presented findings from the Constances study, in which LEQUY et al. [60] investigated the determinants of e-cigarette use, particularly exploring associations with health conditions. Among 18300 participants, who were either ever-users of tobacco or current users of e-cigarettes, $\sim 17 \%$ of current smokers and $\sim 4 \%$ of former smokers used e-cigarettes. The major predictors of e-cigarette use were age, with young adults reporting the highest prevalence, increasing cumulative tobacco consumption and suffering from respiratory conditions. Finally, Marine Gambaryan presented the findings of the Russian Tobacco Control Law Evaluation Survey on the use of e-cigarettes. Among 11699 participants, $12 \%$ had ever used e-cigarettes and almost $26 \%$ of those were regular users [61]. Among cigarette smokers, $\sim 44 \%$ had tried to quit using e-cigarettes but more than two-thirds failed to reduce cigarette consumption. Finally, the study shows that while most participants were against vaping in indoor public and private places, a significant proportion witness vaping in public places and the majority had been exposed to vaping aerosols in public places where smoking is legally banned [61].

\section{Emergent tobacco products and harm reduction: a tobacco solution to a tobacco problem?}

In this Hot Topic session, the focus was on heated tobacco products (HTPs), and the pros and cons of using them. The first speaker, Dimos Fotopoulos, talked about the main role of nicotine addiction in maintaining tobacco consumption, as well as harm reduction principles and its public health applications [62]. Addiction is a chronic phenomenon of brain reward, motivation, memory and related circuitry [63]. Most tobacco products, including HTPs and e-cigarettes, are devices of inhaled nicotine, which is highly addictive and poses challenges to treatment interventions [64]. Some approaches to quitting smoking promote minimisation of risks and harms of tobacco use but without motivation and promotion of a healthy lifestyle, satisfactory health outcomes are difficult to achieve. According to Dimos Fotopoulos, emergent tobacco and nicotine delivery devices with allegedly harm reduction potential are examples of inadequate harm reduction approaches as they do not encourage nicotine cessation and rather stimulate the continuation of nicotine use.

The second speaker, Linnea Hedman, discussed causality in public health science and reviewed the evidence on the potential human health effects of HTPs [65]. Public health advocates have stressed that the short- and long-term health effects resulting from continued consumption of addictive products such 
as HTPs remain unclear. Moreover, given the tobacco industry's long history of deceit about tobacco health hazards [66], there is an urgent need for independent research and robust scientific evidence on these products. To date, few independent studies have explored the components or the emissions of HTPs or studied the health effects of these products in animal models. However, Hedman suggested that scientific uncertainty should not be used as a reason to postpone preventive measures and that the precautionary principle should be applied to stop HTPs from being used.

Elif Dagli talked about industry tactics focusing on harm reduction and the development of novel HTPs [67]. In 2015, the profits of six major tobacco companies reached USD 62 billion. In the same year, 6.4 million people died due to tobacco-related diseases [68]. In an attempt to improve its image, the industry is trying to eliminate cigarettes, replacing them with smoke-free e-cigarettes, and to reduce tobacco-related mortality. They have been funding research, scientific meetings and publications. For example, Philip Morris has committed USD 80 million from 2018 to 2029 to the Foundation for a Smoke-Free World, whose goal is to eliminate smoking worldwide, and advance smoking cessation and harm-reduction science and technology [68-70]. A survey of tobacco industry documents by Stanton Glantz and colleagues showed that the tobacco industry's early HTP pursuits were primarily driven by non-health related reasons, in particular to evade smoke-free regulations and to complement, rather than compete with, conventional cigarettes [71]. Other research of tobacco industry documents by Anna Gilmore and colleagues revealed that tobacco industry's use of harm reduction was aimed to gain access to scientists, the public health community and policy makers, and rebuild its reputation [72]. According to Dagli, HTPs are not risk free, their use could renormalise tobacco use and there is no evidence to suggest they will reduce the use of cigarettes. She concluded that tobacco industry tactics have not changed.

Isabel Saraiva gave a patient's perspective on emerging tobacco and nicotine products [73]. She stressed that smokers suffering from a lung condition need help and support from HCPs to quit. Isabel presented herself as "an ex-heavy smoker" suffering from COPD and stated that when she decided to quit, neither e-cigarettes nor HTPs were on the market. She also said: "I managed to quit for life. I doubt that I would have stopped inhaling nicotine if these products were available. There are neither harmless tobacco nor safe nicotine inhaled products".

The last speaker in this session was Charlotta Pisinger, who presented the WHO and the Tobacco Control Committee (TCC) position on HTPs [74]. The WHO and the WHO Framework Convention on Tobacco Control (FCTC) Secretariat have clearly warned that HTPs are addictive, have been marketed "in pursuit of profit rather than public health", and should be subjected to the same policy and regulatory measures applied to tobacco products, in line with the FCTC [75]. As the leading respiratory medical organisation, ERS works strategically in tobacco control and promotes tobacco cessation, both from a policy and clinical approach [52]. Charlotta Pisinger presented the TCC statement on HTPs and announced that they are preparing another on harm reduction to be submitted for approval by the ERS leadership.

\section{Concluding remarks}

Asthma and COPD are still the two most heavily studied diseases in the field of respiratory epidemiology. Recent findings on the genetics of both diseases have provided us with more questions than answers and have shown that to understand the molecular mechanisms of these diseases, new studies should bring together genetics, epigenetics, transcriptomics, other 'omics and epidemiological methods. Novel areas of research, such as the exposome or the microbiome, could help to further understand the interplay of respiratory disease and lung function with various environmental and lifestyle risk factors. Research on shared genetic and environmental risk factors for the co-occurrence of chronic respiratory disease with other diseases is limited and should be promoted. In addition, findings from studies on new nicotine products and innovative ways to quit smoking emphasise that all tobacco and e-cigarette products are additive and harmful. We hope that this summary of the remarkable amount of research in the field of respiratory disease epidemiology and tobacco control presented through the Epidemiology and Environment Assembly of the ERS creates curiosity to follow up on topics of interest to each reader.

Conflict of interest: M. de Vries has nothing to disclose. E.L. Axson has nothing to disclose. J. Ratanachina has nothing to disclose. O. Dumas has nothing to disclose. S. De Matteis has nothing to disclose. A. Bédard reports ERS Young Scientist sponsorship and an ERS Long Term Fellowship (LTRF 2015-5838), outside the submitted work. C. Roda reports grants from the European Commission/European Respiratory Society outside the submitted work. S. Moitra reports a Long Term Research Fellowship (LTRF 201701-0088) from the European Respiratory Society, ERS Young Scientist 2018 sponsorship, an editorial honorarium from Lancet Respiratory Medicine, faculty remuneration from the European Respiratory Society, an editorial honorarium from Current Respiratory Medicine Reviews and a nonfinancial honorarium from Oxford Publications, outside the submitted work. E. Dagli has nothing to disclose. A. Diamanti has nothing to disclose. A.G. Dilektasli has nothing to disclose. S. Ravara has nothing to disclose. A.F.S. Amaral has nothing to disclose. 


\section{References}

1 Wain LV, Shrine N, Artigas MS, et al. Genome-wide association analyses for lung function and chronic obstructive pulmonary disease identify new loci and potential druggable targets. Nat Genet 2017; 49: 416-425.

2 Wain L, Shrine N, Guyatt A, et al. A weighted genetic risk score based on 279 signals of association with lung function predicts Chronic Obstructive Pulmonary Disease. Eur Respir J 2018; 52: Suppl. 62, OA2188.

3 Oelsner E, Smith B, Nguyen J, et al. Associations between a COPD genetic risk score and lung structure on computed tomography (CT): SPIROMICS. Eur Respir J 2018; 52: Suppl. 62, OA2187.

4 Terzikhan N, Sun F, Verhamme F, et al. Heritability and genome-wide association study of diffusing capacity of the lung (DLCO). Eur Respir J 2018; 52: Suppl. 62, OA2189.

5 Bouzigon E, Veil R, Mohamdi H, Granell R, et al. Influence of gene-by-sex interaction on time-to-asthma onset: a large-scale genome-wide meta-analysis. Eur Respir J 2018; 52: Suppl. 62, OA2190.

6 Minelli C, Van Der Plaat D, Leynaert B, et al. The effect of early puberty on asthma in women and men: a Mendelian randomization study. Eur Respir J 2018; 52: Suppl. 62, OA2192.

7 Bédard A, Northstone K, Holloway JW, et al. Maternal dietary antioxidant intake in pregnancy and childhood respiratory and atopic outcomes: birth cohort study. Eur Respir J 2018; 52: Suppl. 62, OA2191.

8 de Vries M, Van Der Plaat DA, Nedeljkovic I, et al. Identification of novel rare genetic variants associated with COPD in the general population. Eur Respir J 2018; 52: Suppl. 62, OA2193.

9 Chang D, Choy D, Bhangale T, et al. A whole genome sequencing association study of severe, uncontrolled asthma. Eur Respir J 2018; 52: Suppl. 62, OA2194.

10 Backman H, Ekerljung L, Eriksson B, et al. Decrease in prevalence of COPD in Sweden after decades of decrease in smoking. Eur Respir J 2018; 52: Suppl. 62, OA1935.

11 Rabe KF, Watz H. Chronic obstructive pulmonary disease. Lancet 2017; 389: 1931-1940.

12 Kim S, Kim H, Cho N, et al. Identification of FAM13A gene associated with the ratio of FEV1 to FVC in Korean population by genome-wide association studies including gene-environment interactions. J Hum Genet 2015; 60: 139-145.

13 Kim S, Lee KY, Lee SK, et al. The effects of smoking cessation and FAM13A gene susceptibility on lung function decline: A 16-year follow-up study among male smokers. Eur Respir J 2018; 52: Suppl. 62, OA1932.

14 Kaluza J, Larsson SC, Orsini N, et al. Fruit and vegetable consumption and risk of COPD: a prospective cohort study of men. Thorax 2017; 72: 500-509.

15 Meteran H, Thomsen SF, Miller MR, et al. Self-reported intake of fruit and vegetables and risk of chronic obstructive pulmonary disease: a nation-wide twin study. Eur Respir J 2018; 52: Suppl. 62, OA1936.

16 Morgan AD, Zakeri R, Quint JK. Defining the relationship between COPD and CVD: what are the implications for clinical practice? Ther Adv Respir Dis 2018; 12: 1753465817750524.

17 Husebø GR, Gabazza E, D’Alessandro C, et al. Coagulation markers in COPD. Eur Respir J 2018; 52: Suppl. 62, OA1937.

18 Rasmussen D, Bodtger U, Lamberts $\mathrm{M}$, et al. Beta-blocker, aspirin and statin usage after myocardial infarction in patients with and without COPD. A nationwide analysis from 1995 to 2015 in Denmark. Eur Respir J 2018; 52: Suppl. 62, OA1933.

19 GDB 2015 Disease and Injury Incidence and Prevalence Collaborators. Global, regional, and national incidence, prevalence, and years lived with disability for 310 diseases and injuries, 1990-2015: a systematic analysis for the Global Burden of Disease Study 2015. Lancet 2016; 388: 1545-1602.

20 GDB 2015 Mortality and Causes of Death Collaborators. Global, regional, and national life expectancy, all-cause mortality, and cause-specific mortality for 249 causes of death, 1980-2015: a systematic analysis for the Global Burden of Disease Study 2015. Lancet 2016; 388: 1459-1544.

21 Hoyer $\mathrm{N}$, Thomsen $\mathrm{L}$, Wille $\mathrm{M}$, et al. Increase in hospital admissions in smokers with Interstitial Lung Abnormalities in the Danish Lung Cancer Screening Trial (DLCST). Eur Respir J 2018; 52: Suppl. 62, OA1939.

22 Gayle A, Axson EL, Bloom CI, et al. Changing causes of mortality for people with chronic respiratory diseases. Eur Respir J 2018; 52: Suppl. 62, OA1934.

23 De Matteis S, Feary J, Macfarlane J, et al. Update of the British Occupational Health Research Foundation (BOHRF) guidelines on occupational asthma. Eur Respir J 2018; 52: Suppl. 62, OA1950.

24 Henneberger PK, Caridi M, Humann M, et al. Work tasks and occupations as risk factors for asthma in a sample of urban healthcare workers. Eur Respir J 2018; 52: Suppl. 62, OA1951.

25 Dumas O, Le Moual N, Lowe AJ, et al. Influence of childhood asthma and allergies on occupational exposure in early adulthood: a prospective cohort study. Eur Respir J 2018; 52: Suppl. 62, OA1948.

26 Suarthana E, Taghiakbari M, Parfi AA, et al. Effect of welding fumes on rhinitis symptoms and nasal patency: Quebec apprentice cohort study. Eur Respir J 2018; 52: Suppl. 62, OA1952.

27 Kamravaei S, Machichi KI, Miandashti N, et al. Analysis of chronic occupational exposure in non-smoking insulators. Eur Respir J 2018; 52: Suppl. 62, OA1947.

28 Begin R, Bergeron D, Samson L, et al. CT assessment of silicosis in exposed workers. Am J Roentgenol 1987; 148 509-514.

29 Ophir N, Bar Shai A, Korenstein R, et al. Functional, inflammatory and imaging impairments due to artificial stone dust (ASD) ultrafine particles(UFP) exposure. Eur Respir J 2018; 52: Suppl. 62, OA1953.

30 Hessel PA, Goldstein B, Davies JC, et al. Pathological findings in mine workers: I. Description of the PATHAUT database. Am I Ind Med 1987; 12: 71-80.

31 Murray J, Ndlovu N, Richards GA. Evaluation of autopsy data for occupational lung disease surveillance in South African miners. Eur Respir J 2018; 52: Suppl. 62, OA1954.

32 Thompson E, Powell P, Heederik D, et al. Using digital and social media to highlight the risks of occupational exposures as a cause of lung diseases. Eur Respir J 2018; 52: Suppl. 62, OA1949.

33 Oelsner E, Balte P, Bhatt S, et al. Accelerated lung function decline in former and light smokers: NHLBI Pooled Cohorts. Eur Respir J 2018; 52: Suppl. 62, OA5179.

34 Johannessen A, Nordeide Kuiper I, Accordini S, et al. Long-term air pollution exposure is associated with sick leave 20 years later. Eur Respir J 2018; 52: Suppl. 62, OA5182.

35 Carlsen HK, Nyberg F, Segersson D, et al. Chronic exposure to black carbon particles and traffic-related particulate matter (PM) specifically from traffic are associated with reduced lung-function in a general population. Eur Respir J 2018; 52: Suppl. 62, OA5186. 
64 Benowitz NL. Nicotine addiction. N Engl J Med 2010; 362: 2295-2303.

65 Hedman L. Health effects of heated tobacco products: what we do and do not know. Eur Respir J 2018; 52: Suppl. 62,4912

66 Malone RE, Chapman S, Gupta PC, et al. A "Frank Statement" for the 21st Century? Tob Control 2017; 26: 611-612.

67 Dagli E. How tobacco industry tactics have changed over time: effective regulation is needed. Eur Respir J 2018; 52: Suppl. 62, 4913.

68 Drope J, Schluger N, Cahn Z, et al. Manufacturing. In: The Tobacco Atlas. https://tobaccoatlas.org/wp-content/ uploads/2018/03/TobaccoAtlas_6thEdition_LoRes_Rev0318.pdf

69 Campaign for Tobacco-Free Kids. If Philip Morris is serious about a "Smoke-Free World," it should stop marketing cigarette, Fighting efforts to reduce smoking. https://www.tobaccofreekids.org/press-releases/2017_09_ 13_pmi

70 WHO. WHO Statement on Philip Morris funded Foundation for a Smoke-Free World. www.who.int/en/newsroom/detail/28-09-2017-who-statement-on-philip-morris-funded-foundation-for-a-smoke-free-world

71 Dutra LM, Grana R, Glantz SA. Philip Morris research on precursors to the modern e-cigarette since 1990. Tob Control 2017; 26: 97-105. 
72 Peeters S, Gilmore AB. Understanding the emergence of the tobacco industry's use of the term tobacco harm reduction in order to inform public health policy. Tob Control 2015; 24: 182-189.

73 Saraiva I. Patient perspectives on emerging tobacco and nicotine products. Eur Respir J 2018; 52: Suppl. 62, 4914.

74 Pisinger C. WHO's and the tobacco control committees' position on harm reduction and emerging tobacco products. Eur Respir J 2018; 52: Suppl. 62, 4915.

75 WHO. Tobacco Free Initiative. Heat-not-burn tobacco products information sheet. www.who.int/tobacco/ publications/prod_regulation/heated-tobacco-products/en/ 